\title{
PREDICTING THE HUMAN RESPONSE TO AN EMERGENCY
}

\author{
G. Lawson ${ }^{1}$, S. Sharples ${ }^{1}$, S. Cobb ${ }^{1}$ and D. Clarke $^{2}$ \\ 1. Human Factors Research Group, University of Nottingham, UK \\ 2. School of Psychology, University of Nottingham, UK
}

\begin{abstract}
This paper presents a case study of an approach for predicting the human response to a domestic fire, using a combination of a talk-through technique (Kirwan and Ainsworth, 1992) and sequential analysis (Bakeman and Gottman, 1986). 20 participants were asked what actions they would take upon hearing a strange noise in their house, which they were later told was a fire. Each act was recorded and the results were compared to previous research in which people involved in real fires had been interviewed (Canter et al, 1980). A significant relationship was found between the frequency (Spearman's rho: 0.694, p<0.01) and sequence (Spearman's rho: $0.441, \mathrm{p}<0.05$ ) of acts in this study and those from the interviews with people involved in real fires. More work is needed to develop the approach, but this case study indicates that it might have use as a low-cost method which can be used to predict behaviour in an emergency.
\end{abstract}

\section{Introduction}

There is little guidance for ergonomists attempting to predict the human behavioural response to an emergency such as a building fire or an industrial explosion. Predicting behaviour in an emergency has several useful applications, for example: populating simulation tools which can be used for training emergency responders; investigating alternative factory or building layouts for emergency evacuation planning; or planning for crowd safety and the response to major emergencies. Basing predictions on previous events is not always possible as gaining access to data such as incident reports can be difficult, and the behaviours exhibited may be specific to the circumstances in which the event occurred. Laboratory studies are also rightly constrained by ethics considerations which prevent putting participants in a dangerous or distressing situation. Laughery (2005) recognises the importance of predicting human performance, and has provided excellent guidance for giving quantitative estimates to support simulating and modelling human performance. He gives examples of a predictive comparison of interaction methods and of predicting the workload demands for driving a car. However, his methods are not obviously applicable to understanding what actions people will take when placed in an emergency, as they 
require some knowledge of the tasks people will perform or models of human performance before they can be used. Nevertheless, they may be useful when attempting to quantify or analyse behaviours identified through other methods.

Attempts to predict human behaviour are reported in computer simulation literature, as developers of software tools use these predictions to programme computer representations of people to increase the realism of their simulations. An approach used by several developers of simulation models is to research published models of human behaviour, then implement these as code in their simulation tools (Cornwell et al, 2002, Silverman et al, 2006, Pan et al, 2006, Baines et al, 2005). However, this approach has been described as difficult for the following reasons: published models of behaviour can be unspecific, un-quantified or incomplete; insufficient integration exists between different areas of research; developers have insufficient knowledge and understanding of psychology and behaviour; and poor communications exist between people working in social sciences and computer programming (Silverman et al, 2001, Silverman et al, 2006).

This study is an initial investigation towards a new approach for behaviour prediction. The approach draws from existing methods used for studying and analysing behaviour. Sequential analysis (Bakeman and Gottman, 1986) is an established method for studying behaviour, which involves the study of events or interactions as they unfold over time, and is therefore particularly suited to studying dynamic aspects of behaviour. The basic method is to record behavioural sequences using a taxonomy of behaviours. For example, if a person displays behaviour $\mathrm{X}$, this is recorded. The type of behaviour displayed subsequently (e.g. Y) is also recorded, and so on for a sample of people, for any task/scenario of interest. This data can be used to identify recurrent sequences of behaviours, and the probability of one particular type of behaviour following another (e.g. the probability that behaviour $\mathrm{Y}$ will follow behaviour $\mathrm{X}$ ). If the results of the analysis are represented in diagrammatical format they can demonstrate the flow of different behaviours and the probability of moving from one to another. Furthermore, generic behaviours can then be abstracted from the analysis.

This technique has already been applied to investigate patterns of behaviour in domestic, multiple occupancy and hospital fires (Canter et al, 1980). A team of researchers recognised that fire safety regulations were based upon invalidated assumptions of behaviour in a fire and therefore attempted to gain more detailed and empirical data. Initially, they obtained information from the fire brigade regarding the occurrence of different types of fires. They then attempted to gain statements from witnesses involved in the fires that had occurred, supplementing this with information from press reports and police witness statements. The witnesses were asked to describe exactly what they did from the time they noticed something abnormal was happening, until after they exited the building. These descriptions were transcribed and were coded against a taxonomy of behaviours. The researchers then recorded the frequency with which each of the behaviours followed every other behaviour. From this they generated decomposition diagrams demonstrating the probability of moving to any one of the behaviours from any other stage in the sequence. Finally, they attempted to extract generic models of behaviour which were displayed in all types of residency studied. Thus, sequential analysis was used as a method for modelling and predicting behaviour in emergency situations. The approach presented in this paper aims to yield the same type of sequential analysis as in the work by Canter et al (1980), but using individuals' predictions of their likely behaviour in a fire rather than actual data from those who have experienced fires. However, sequential analysis must be applied to observable or recordable behaviours; as a technique it only describes and analyses behaviour, it does not generate any behavioural phenomena. For this study, a talkthrough method was selected as an appropriate method of generating the behaviours of interest. 
In this method, respondents are simply asked to describe their actions in response to a scenario or statement. It is a low-cost approach, as no special equipment is required (Kirwan and Ainsworth, 1992). Furthermore, using the talk-through technique meant that a dangerous situation could be described and participants' responses, feelings and opinions could be recorded without putting them in actual danger. As this method is low cost and quick to implement, it was chosen for this study in lieu of observable or reportable behaviour in a real fire.

\section{Method}

The method was selected such that the study described above (Canter et al, 1980) could be used to validate the results from this case study. 20 participants ( 10 male; 10 female; mean age $=36$, range $=20-52)$ were recruited from staff and students of the University of Nottingham. Participants were allocated a one hour appointment. They were asked to sketch a plan layout of their house, and identify who would typically be in their house at night-time. Participants were then asked to detail, in order, every action they would take after hearing a faint cracking noise coming from their kitchen, which on investigation they were told was a fire. They were told to stop their description when they reached the point where they imagined they would be completely out of danger, typically upon exiting their house. If their responses contained insufficient detail, or if they missed a logical stage, they were probed with statements such as "I think you're missing something there" or "I'd like more detail about the stages between those acts". Their stated acts were recorded using a laptop computer, which the participants were able to check as it was projected onto a display screen. The sketches of the participants' plan views of their houses were also recorded. The whole experiment was conducted with procedures which received approval from the University of Nottingham Faculty of Engineering Ethics Committee.

The example used was informed by a number of assumptions. First, it was assumed that the fire started in the kitchen as anecdotal evidence suggests that this is where most fires begin (e.g. Bosley, 2003). Participants were only told they could hear the smoke alarm upon entering the kitchen. This second assumption was unsupported by data from real fires, but prevented participants from stating they would exit the property as soon as they heard a fire alarm, a sequence of events which was experienced in the pilot studies but was not supported by Canter et al (1980), probably due to an increase in the prevalence of smoke alarms since the original study. If participants stated that they would fight the fire, they were told that despite their attempts, the fire would not be put out. Also, if participants reported that they would send someone else to investigate the noise, they were told that this person informed them that there was a fire upon their return.

The actions described by the participants were coded using the taxonomy of acts generated by Canter et al (1980). Where participants described an act which could not reliably be mapped to an act in the original study, new codes and activities were created. The coding of participants' behaviours was reviewed by an independent researcher to check for consistency.

A matrix was then created in which the number of times each act followed every other act was recorded. The matrix was used to generate standard residuals for each transition (observed frequency minus expected frequency, divided by square root of expected frequency). Canter $e t$ al (1980) reported these values as "strength of association" and used them to provide information on the relationships between acts on decomposition diagrams (see Figure 2). 


\section{Results}

Initially, the 49 acts from the original Canter et al (1980) study were scrutinised, and any which would not have been expected in this case study due to the example scenario were omitted from the analysis. These were mainly related to actions for which insufficient detail was reported in the original study, and therefore to use them in this study would have introduced inaccuracies. For example: any tasks regarding involvement with smoke were omitted as there was no data on the spread of smoke in Canter et al (1980); any act in response to another person, aside from a partner returning after investigating the fire, was omitted as again no data was published in the original study on the actions of the other people; struggling with fire equipment and any action relating to someone who was not from the house containing the fire were also omitted. After this process, 23 of the 49 acts originally described by Canter et al (1980) were deemed suitable for comparison. This corresponded to a total of 233 statements (or 65\%) of all 361 statements reported in this experiment; 34 statements $(9 \%)$ were excluded as a result of the process described above, the other 94 statements $(26 \%)$ were reported in this experiment but could not be mapped onto acts in the original taxonomy.

A scatter plot of the results, showing the frequency of comparable acts from the original study against those from this experiment is shown in Figure 1.

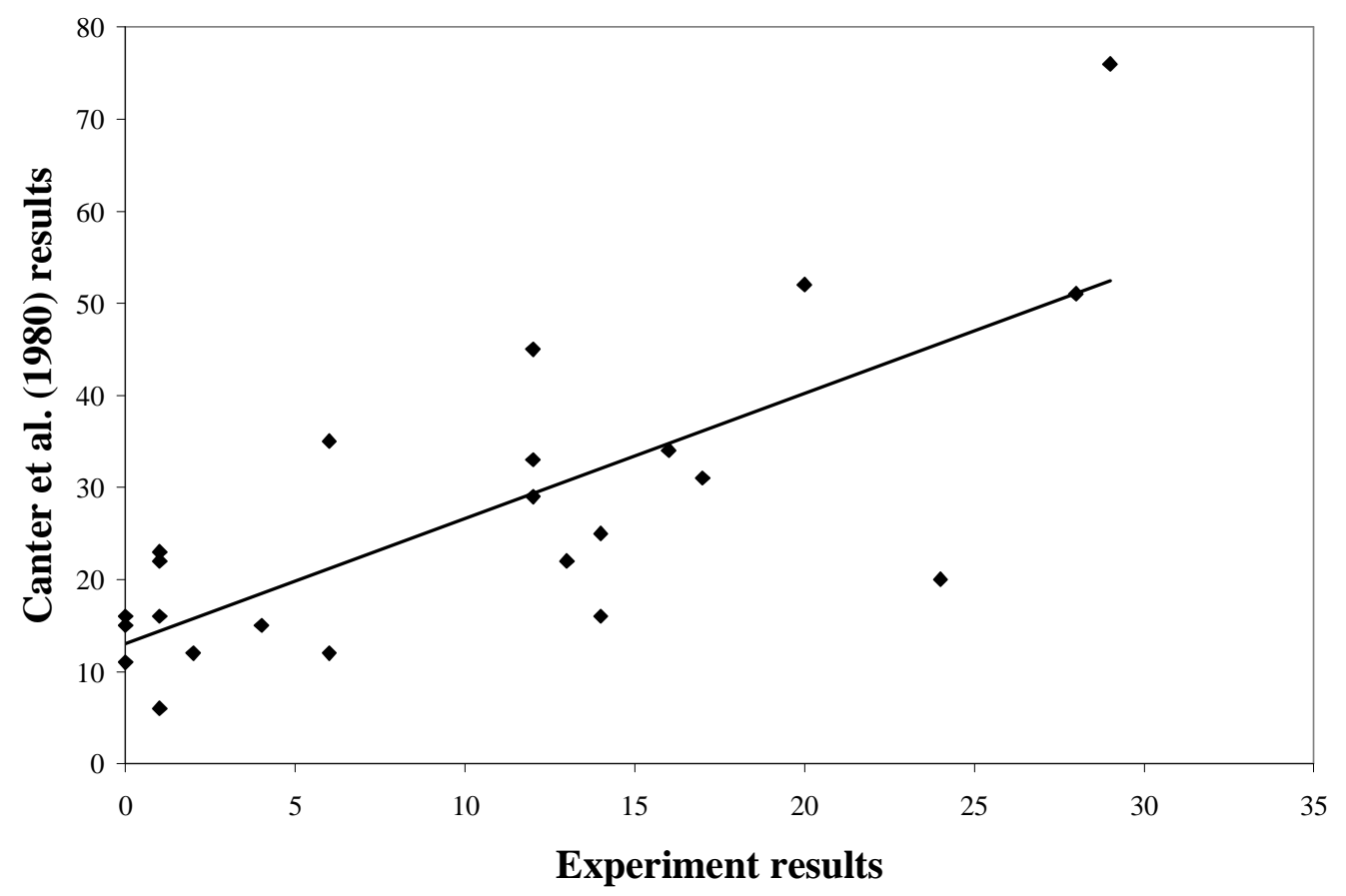

Figure 1. Comparison of the frequency of 23 acts from Canter et al (1980) to corresponding acts from this experiment

A Spearman's rho test demonstrated that a significant relationship existed between the frequency of acts in the original study which were compared to those from this experiment (Spearman's rho: 0.694, $\mathrm{p}<0.01$ ).

The transition matrix was used to investigate the sequence of actions. The standard residuals were identified for any sequences between the 23 comparable acts for which values were 
reported in Canter et al (1980). For these transitions, the correlation between the standard residuals reported in Canter et al (1980) and from this study was found to be significant (Spearman's rho: $0.441, \mathrm{p}<0.05$ ). The values used in the comparison are shown on a decomposition diagram in Figure 2. The strength of association values from Canter et al (1980) are labelled on arrows which point to subsequent acts; the corresponding values from this experiment are shown in brackets. There is no meaning in the position or proximity of the nodes.

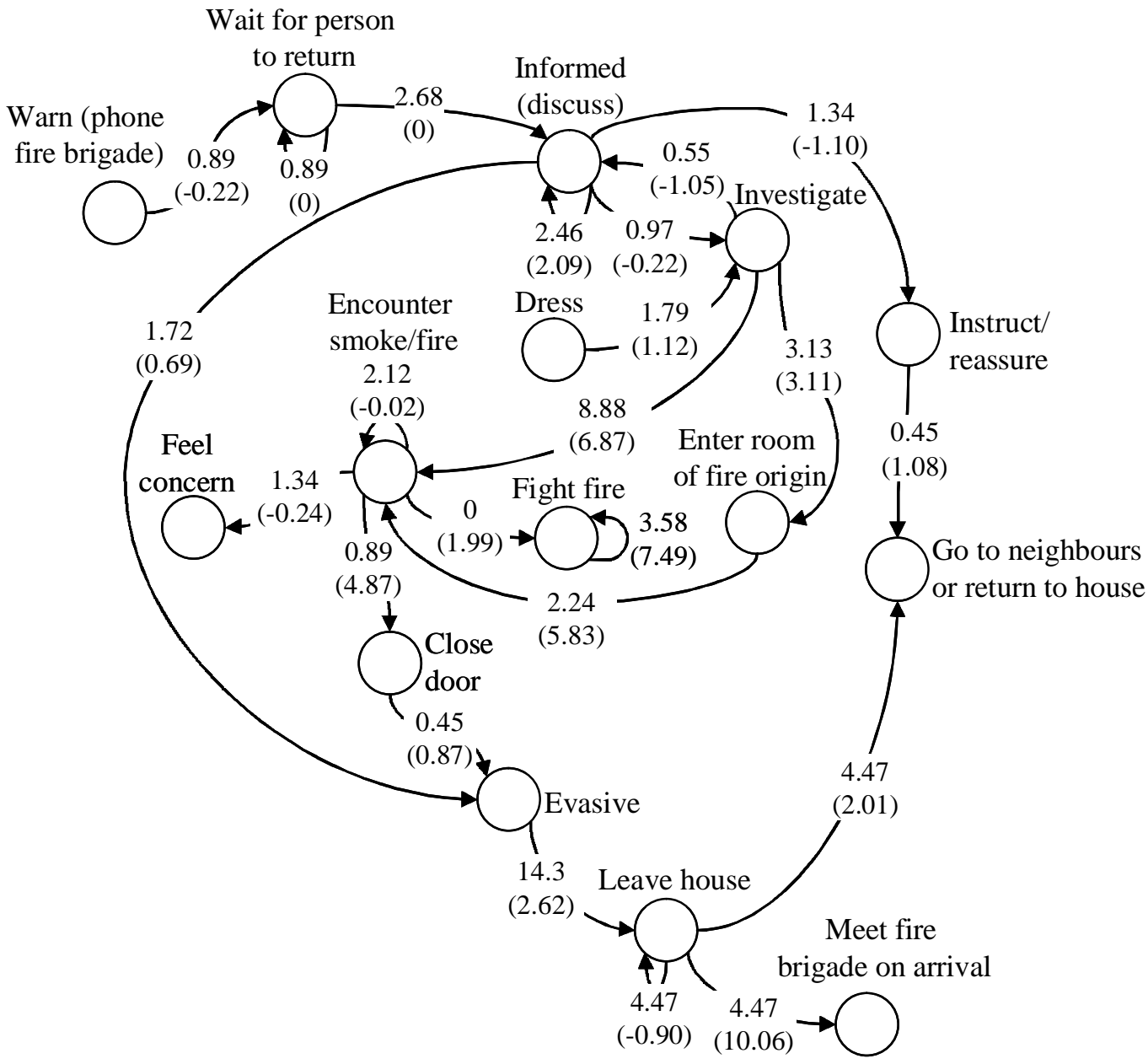

Figure 2. Decomposition diagram showing strength of association values from Canter et al (1980), and those from this experiment in brackets

\section{Discussion}

In this instance, the technique demonstrated a statistically significant relationship between the frequencies of acts found in this study with those from the original interviews with survivors of real fires. The sequences of the acts also demonstrated a statistically significant relationship with those from the original study. Therefore, in this example, a predictive approach was able to 
provide an indication of how people will behave in a domestic fire. It was achieved with minimal resources; 20 people each giving up to one hour in a basic office meeting room with a laptop, projector and whiteboard.

There are aspects of the predictive approach which require further development. In particular, the validity needs to be assessed. Although face validity of this approach appears to be low sitting comfortably in an office environment during the day is very different to be being woken by a fire in a home at night - the approach did produce a reasonable prediction of what actions people will take, when measured against the behaviour of people in actual domestic fires. However, this study only demonstrates comparability with another study. If access was gained to a more detailed incident report from an emergency scenario, this would allow for a more finegrained analysis of the behaviours, and would also permit inclusion of some of the acts omitted from this study, such as interaction with smoke. A more detailed account of human behaviour in fire would also allow inclusion of timing of acts, as well as frequencies and sequences.

The generalisability and reliability of the technique cannot be evaluated based on this case study alone: it would need to be repeated and applied to other types of emergency situations. The Canter et al (1980) study provides results from multiple occupancy and hospital fires for which this technique could be applied to investigate the generalisability. Work is already underway to begin investigating the reliability by repeating this study with a further 20 participants.

Another limitation of the study is that performance influencing factors such as time-of-day effects, fatigue and training have not been accounted for. It is acknowledged that these are likely to influence behaviour, particularly in an industrial setting. However, the study used for comparison of results (Canter et al, 1980) had no information regarding these factors. Therefore to include them in this study would have introduced confounding variables. They should, however, be investigated as part of the future development of this approach.

The approach only reveals what actions the respondents predict they would do; it does not reveal the causes of their behaviours. That is, it attempts to answer the question "what would people do?" not "why would they do it?" Obviously the latter would be of interest to an ergonomist when attempting to fully understand the behaviours, and how they might be influenced by workplace design or training.

Despite the limitations and further work required, in this example the approach was able to predict many of the behaviours people may demonstrate in a domestic fire, and gave an indication of their sequences. Although it does not identify or provide an understanding of the reasons why people exhibited these behaviours, it was a low-cost and efficient method, which did not rely on experts or people who have experienced a fire. Therefore, it is anticipated that it could have use as a "first-pass" method for predicting behaviour in an emergency, which could then be supplemented by other more involved approaches. For example, the results from this study could be used to increase the realism of the computer representations of people in a simulation tool by incorporating probabilities of various behaviours. The simulation could then be reviewed and further refined through other methods to increase its validity.

\section{Conclusions}

This case study examined the use of a new approach to predicting human behaviour in a domestic fire. The technique was low cost and was conducted quickly, yet in this example still gave reasonable results for the frequency and sequences of acts when compared to another study based on past events. Therefore, it might have use as a method for predicting behaviour in new situations for which there is no existing knowledge. However, further work is required on the 
method, in particular to test reliability and generalisability and to develop greater confidence in the validity of the results. With development, there is potential for this approach to offer a variety of uses for understanding how people will behave in an emergency situation. For example, in an industrial setting, this approach could be used to generate information that could help develop appropriate response plans, evacuation procedures, signage, training programmes or building layouts. It is anticipated that despite the short-comings, the approach could be used to quickly obtain indications of how people might behave, with minimal resources.

\section{References}

Baines, T. S., Asch, R., Hadfield, L., Mason, J. P., Fletcher, S. and Kay, J. M. 2005, Towards a theoretical framework for human performance modelling within manufacturing systems design. Simulation modelling practice and theory, 13, 486 - 504

Bakeman, R. and Gottman, J. M. 1986, Observing Interaction: An Introduction to Sequential Analysis, (Cambridge University Press, Cambridge)

Bosley, K. 2003, Domestic Firefighting. Accessed on World Wide Web January 2008. Available from: http://www.rmd.communities.gov.uk/project.asp?intProjectID=10887.

Canter, D., Breaux, J. and Sime, J. 1980, Domestic, Multiple Occupancy, and Hospital Fires. In D. Canter (ed.) Fires and Human Behaviour. (John Wiley and Sons, New York).

Cornwell, J. B., Silverman, B. G., O'Brien, K. and Johns, M. 2002, A Demonstration of the PMF-Extraction Approach: Modeling The Effects of Sound on Crowd Behavior. In Proceedings of the Eleventh Conference on Computer Generated Forces and Behavioral Representation. (Orlando).

Kirwan, B. and Ainsworth, L. K. 1992. A Guide to Task Analysis (Taylor and Francis, London)

Laughery, R. 2005, Simulation and modelling for analysing human performance. In J.R. Wilson and N. Corlett (eds.) Evaluation of Human Work, Third Edition, (Taylor and Francis, London).

Pan, X., Han, C. S., Dauber, K. and Law, K. H. 2006, Human and social behavior in computational modeling and analysis of egress, Automation in Construction, 15, 448-461

Silverman, B. G., Johns, M., Cornwell, J. and O'Brien, K. 2006, Human Behaviour Models for Agents in Simulators and Games: Part I - Enabling Science with PMFserv, Presence: teleoperators and virtual environments, 15, 139-162

Silverman, B. G., Might, R., Dubois, R., Shin, H., Johns, M. and Weaver, R. 2001, Toward a Human Behaviour Models Anthology for Synthetic Agent Development. In 10th CGF Proceedings, (IEEE, New York)

\section{Acknowledgements}

The authors acknowledge Professor David Canter and John Wiley \& Sons Ltd for permission to use and modify material from Domestic, Multiple Occupancy, and Hospital Fires (Canter et al 1980). 